\title{
Septum en Seno Maxilar. Un Estudio de 91 Cirugías Consecutivas de Elevación de Piso Sinusal
}

\author{
Septum on Maxillary Sinus. A Study in 91 Consecutive \\ Surgeries of Maxillary Sinus Floor Elevation
}

\author{
"Sergio Olate; "* Leandro Pozzer; ${ }^{* * *}$ Aníbal Henrique Barbosa Luna; **Marcio de Moraes; \\ *** Renato Mazzonetto; ${ }^{* *}$ Roger W. Fernandes Moreira \& ** Jose Ricardo de Albergaria-Barbosa
}

OLATE, S.; POZZER, L.; LUNA, A. H. B.; MORAES, M.; MAZZONETTO, R.; MOREIRA, R. W. F. \& ALBERGARIABARBOSA, J. R. Septum en seno maxilar. Un estudio de 91 cirugías consecutivas de elevación de piso sinusal. Int. J. Morphol., 29(4):1219-1222, 2011.

RESUMEN: La elevación de piso sinusal maxilar ha sido ampliamente discutida y presenta altas tasas de éxito. El objetivo de esta investigación fue establecer la prevalencia de septum intrasinusal presente en cirugías consecutivas de elevación de piso sinusal y establecer su relación con las perforaciones de membrana sinusal. Noventa y un cirugías fueron realizadas en pacientes que presentaban indicación para instalar implantes dentales en maxila posterior; fueron incluidos aquellos pacientes que presentaron documentos clínicos íntegros (ficha clínica, radiografías, entre otros) y que no presentasen contraindicación del procedimiento; las cirugías fueron realizadas con anestesia local o general y fueron rellenados con biomateriales o hueso autógeno. El septum fue identificado mediante la imagen panorámica preoperatoria y de forma clínica intraoperatoria, mientras que cualquier complicación fue estudiada y relacionada con la presencia de septum; el estudio estadístico se realizó a través de la prueba test de Fisher siendo reconocido el valor de p<0.05. Fueron operados 72 sujetos (53 mujeres y 19 hombres) con una edad promedio de 44,5 años; en el 74,7\% no se observó ningún tipo de complicación, mientras que en el 18,7\% de los casos se observó perforación de la membrana sinusal. Septum sinusal fue observado en el 17,6\% de los casos operados (16 senos maxilares), presentando 12 septum en posición anterior y 4 en posición posterior. La presencia de septum tuvo relación estadísticamente significativa con la laceración de membrana sinusal ( $<<0,05)$. Finalmente, podemos señalar que la presencia de septum intrasinusal se observó en $17 \%$ de los casos y se relaciona directamente con complicaciones intraoperatorias como la perforación de membrana.

PALABRAS CLAVE: Seno maxilar; Septum maxilar; Reconstrucción ósea.

\section{INTRODUCCIÓN}

La rehabilitación oral del sector posterior de maxila muchas veces requiere aumento óseo o modificaciones de diseño de implantes que permitan insertarlos en lugares anatómicamente disminuidos (Raghoebar et al., 1997; Kim et al., 2011), ya que luego de la perdida dentaria del sector posterior de maxila es posible observar pneumatización del seno maxilar y consecuentemente reabsorción ósea alveolar.

Las técnicas quirúrgicas de elevación de seno maxilar han sido descritas con anterioridad y demuestran previsibilidad y éxito en el tratamiento (Boyne \& James 1980). De esta forma, la cirugía puede ser realizada con anestesia local y con la incorporación de diferentes rellenos óseos de forma particulada o en bloque (Jang et al., 2010; Wheeler, 1997; Block \& Kent, 1997).

La cirugía con ventana ósea lateral presenta un acceso quirúrgico similar al acceso de la cirugía de CaldwellLuc, donde a través de una osteotomía se llega hasta la membrana sinusal, la que es descolada y desplazada hacia una posición superior que permite la inserción de algún material de relleno (Boyne \& James). La etapa de descolamiento de la membrana es una de las más críticas debido a que gran parte de las complicaciones asociadas a la laceración de membrana sinusal se producen en ese momento.

* Departamento de Odontología Integral, Universidad de La Frontera, Temuco, Chile

** Departamento de Diagnóstico Oral, Universidad Estadual de Campinas, Campina, Brasil.

**** Departamento de Clínica y Odontología Social, Universidad Federal de Paraíba, João Pessoa, Brasil. 
La presencia de variaciones óseas intrasinusales fue descrita inicialmente por Underwood en el año 1910 en su trabajo clásico, donde destaca la morfología y fisiología del seno maxilar, su desarrollo y sus condicionantes para el desarrollo de patologías (Underwood, 1910); sin duda que la presencia septum intrasinusal y otras variaciones anatómicas puede dificultar la cirugía (Zijderveld et al., 2008), limitando la efectividad del procedimiento. Existen algunos estudios que señalan la presencia de septum intrasinusal en 111 de 400 senos maxilares evaluados mediante tomografía cone beam, señalando también que la posición media fue la más prevalente $(45,9 \%)$ seguida de la región posterior (31,5\%) (Park et al. 2011). Por otra parte, el estudio clínico de Zijderveld et al. en 100 cirugías consecutivas de elevación de seno maxilar evidenció la presencia de $48 \%$ de septum sinusal, señalando las complicaciones asociadas a la técnica de eversión de la pared lateral del seno maxilar debido a la presencia de esta lamina ósea perpendicular que impide el movimiento hacia el interior. Finalmente el estudio de 60 senos maxilares en 30 cadáveres de Rosano et al. (2010) señalo la presencia de un 33\% de senos maxilares con septum intrasinusal.

El objetivo de esta investigación fue establecer la presencia de septum intrasinusal en 91 cirugías consecutivas de elevación de seno maxilar.

\section{MATERIAL Y MÉTODO}

La presente investigación corresponde a un estudio retrospectivo en 72 pacientes operados en el Servicio de Cirugía Oral y Maxilofacial de la Facultad de Odontología de Piracicaba de la Universidad Estadual de Campinas, Brasil. En 91 senos maxilares fue desarrollada la cirugía de elevación de seno maxilar. Fueron incluidos en este estudio todos los pacientes cuyas fichas clínicas se encontraban totalmente completas, con estudios de imagen preoperatorios y postoperatorios, y que se sometieron a cirugía con anestesia local o general. Todos los pacientes que presentaban alguna sintomatología asociada a patología sinusal fueron estudiados, evaluados y tratados por el equipo de otorrinolaringología a fin de limitar las complicaciones asociadas y no operar a quienes presentaran contraindicación.

El estudio pre quirúrgico fue realizado con estudio de imagen panorámica, identificando morfología y características sinusales; también fue solicitado estudio con tomografía computadorizada y con telerradiografía en norma lateral para situaciones más complejas; los modelos de estudio también fueron utilizado para establecer espacio protésico y otras características de la rehabilitación.
Procedimiento quirúrgico. A todos los pacientes les fue administrado $1 \mathrm{~g}$ de amoxicilina y $4 \mathrm{mg}$ de dexametasona 1h antes de la cirugía (vía oral); en casos de cirugía con anestesia general fue administrado $1 \mathrm{~g}$ de cefazolina $\mathrm{y}$ $500 \mathrm{mg}$ de hidrocortisona al inicio del procedimiento quirúrgico. Todos los casos se acompañados de administración de $5 \mathrm{ml}-8 \mathrm{ml}$ anestésico de lidocaína (1:100.000) con vasoconstrictor.

Se realizó una incisión sobre la cresta ósea alveolar, levemente palatinizada, con bisturí № 15 , estableciendo una descarga en el sector posterior y anterior a fin de poder descolar el colgajo mucoperiostico de espesor total. Con motor de baja rotación $(20.000 \mathrm{rpm})$ y fresa cilíndrica diamantada 702 fue realizada una osteotomía lateral, manteniendo irrigación con suero fisiológico $0,9 \%$ constante.

El limite anterior del seno maxilar fue determinado en la radiografía panorámica; a partir de este punto, $5 \mathrm{~mm}$ posterior fue realizada la primera osteotomía vertical y $10 \mathrm{~mm}-15 \mathrm{~mm}$ posterior fue realizada la segunda osteotomía vertical; el límite inferior se estableció en el límite del piso sinusal o $3 \mathrm{~mm}$ superior a el, uniendo ambas osteotomías verticales; el límite superior se estableció a 10mm superior del límite inferior. Una vez terminadas las osteotomías, gentilmente se procedió a descolar la membrana sinusal de la pared lateral y el piso con cucharetas anguladas y rectas de diferentes tamaños. Una vez desplazada la membrana se procedió a incorporar el relleno sinusal con el material seleccionado y sin uso de membrana externa. La sutura fue realizada con seda 3-0 en aguja traumática de $17 \mathrm{~mm}$ y $3 / 4$ de ciclo. En casos de perforación de membrana, cuando esta fue extensa, el procedimiento fue abortado y se sometió a nueva cirugía 6 meses después; en casos de perforaciones menores que $5 \mathrm{~mm}$ se instaló una membrana de colágeno en el área de la perforación o bien se realizó una sutura del lugar respectivo con hilo cat-gut 3-0 en aguja traumática de $17 \mathrm{~mm}$.

Estudio de presencia de septum intrasinusal. Con apoyo de la radiografía panorámica se estableció la presencia de septa y septum intrasinusal que fue posteriormente confirmado durante la cirugía de elevación de seno maxilar. El septum fue medido verticalmente en la radiografía panorámica y fue establecida su posición anterior, media o posterior; además fue establecida su relación con las perforaciones de la membrana sinusal.

Análisis de datos. A través del software Microsoft Excel fue realizado un análisis descriptivo de las variables estudiadas y cuando fue indicado se utilizó el paquete estadístico Stata 9.0 con la prueba test de Fisher siendo reconocido el valor de $\mathrm{p}<0.05$ 


\section{RESULTADOS}

Fueron operados 72 pacientes, siendo 53 sujetos $(73,6 \%)$ del sexo femenino y 19 sujetos del sexo masculino (26,4\%). La edad de los pacientes se encontró en un promedio de 44,5 años (18 a 66 años). De los 72 pacientes, 19 fueron sometidos a cirugía bilateral completando un total de 91 cirugías de elevación de seno maxilar, donde 11 cirugías se acompañaron de injertos óseos de tipo onlay lateral de maxila, fijada con tornillos de osteotsíntesis del sistema 2.0.

El 74,7\% de los senos operados (68 cirugías) se desarrollaron sin complicaciones en el intra o post operatorio. La complicación más frecuentemente encontrada fue la perforación de la membrana sinusal, presente en 17 cirugías (18,7\%), de las cuales 4 cirugías correspondían a un segundo procedimiento luego de haber ocasionado perforación de la membrana durante el primer procedimiento de elevación de seno maxilar.

La presencia de septum intrasinusal fue determinada en 17,6\% de los casos operados (16 senos maxilares). En 12 senos maxilares estos se presentaban en posición anterior y 4 en posición mediana. En dos senos maxilares se observaron dos septa intrasinusal. La altura promedio de los septum fue de $5,8 \mathrm{~mm}$ en la región anterior y 4,6mm en la región mediana. La presencia de septum intrasinusal fue asociado significativamente con la perforación de membrana sinusal $(\mathrm{p}<0,05)$

\section{DISCUSIÓN}

La presencia de septum intrasinusal se ha estudiado de forma anatómica en cadáveres y también a través de radiografías panorámicas y tomografías computadorizadas; dentro de los métodos de imagen, la tomografía computadorizadas ofrece estadísticamente mejores posibilidades diagnosticas (Maestre-Ferrin et al., 2011), determinando la necesidad de estos exámenes para estudios de planificación quirúrgica. Casi 14 años antes, Krenmair et al. (1997) estudiaron 65 cirugías de elevación de seno maxilar evidenciando un $27,7 \%$ de septum, siendo que la imagen panorámica falló en realizar la identificación en el 21,5\% de los casos. En nuestra opinión, esta situación podría estar parcialmente asociada a la calidad de la radiografía más que a al proceso diagnóstico propiamente tal.

Ella et al. (2008) estudiaron 150 senos maxilares, 80 a través de disección anatómica y 70 mediante tomografía computadorizada. El $61 \%$ no presentaba septum o si lo presentaba fue menor que $4 \mathrm{~mm}$. Maestre-Ferrin et al. también señalaron que la mayoría de los septum se encontraban en la región mediana del seno maxilar, con un promedio de altura de $4 \mathrm{~mm}$.
Similares resultados se obtuvieron en nuestra investigación, donde se observaron septum de $5,8 \mathrm{~mm}$ en el sector anterior y $4,6 \mathrm{~mm}$ en la región mediana. Rosano et al. también presentaron un estudio en cadáveres donde observaron que de los 60 senos estudiados, 20 presentaban septum intrasinusal, siendo que ninguno de ellos fue de tipo completo; la gran parte de estos septum se ubicaron de forma vertical en el sector anterior (30\% de los casos) y mediano (40\% de los casos).

Krenmair et al. (1999) estudiaron 194 senos maxilares y concluyeron que en senos maxilares edéntulos la prevalencia de septum se sitúa entre el $27 \%$ y $31 \%$; los senos dentados, por otra parte, presentaban cerca de un $14 \%$ de incidencia de septum. Los autores sugieren la presencia de septum secundarios o del desarrollo que se presentarían posterior a la pérdida dentaria; sin embargo, concordamos con Stover (1999), quien señala que más probable que septum secundario, estos elementos pueden ser remanentes de los alveolos dentarios que se mantienen luego del proceso de reabsorción alveolar y pneumatización del seno maxilar.

Zijderveld et al. en 100 cirugías de elevación de seno maxilar evidenciaron un $48 \%$ de septum intrasinusal, cerca de un 30\% mayor que nuestros resultados; en esta secuencia de pacientes solo 9 presentaron perforación de membrana, siendo 5 asociados a la presencia de septum, demostrando que el uso de dos accesos quirúrgicos puede ser ventajoso para la disminución de esta complicación. En nuestra investigación, la presencia de septum fue directamente asociado a la perforación de membrana, lo cual puede estar asociado a la curva de aprendizaje y experiencia del operador toda vez que fueron 91 cirugías realizadas de forma consecutiva presentando mayores problemas durante la etapa inicial de esta investigación. La laceración o ruptura de la membrana sinusal puede generar complicaciones postoperatorias o retardar el procedimiento, con lo que implica desarrollar un segundo procedimiento quirúrgico aumentando el estrés del paciente y los costos del mismo. Siendo así, reconocer la presencia de septum intrasinusal es fundamental en la planificación quirúrgica; además de eso se debe establecer las condiciones morfológicas de posición y medida del septum para disminuir los riesgos del procedimiento. De hecho, Beltrán et al. (2011) sugieren que el uso de endoscopio para el abordaje en cirugía de seno maxilar podría llevar a un reconocimiento precoz del septum intrasinusal y con ello disminuir las posibles complicaciones; sin embargo, los costos asociados pueden ser una limitante en algunos centros asistenciales.

De esta manera, cuando el septum se presenta de forma vertical con extensiones mayores que $4 \mathrm{~mm}$, la aproximación quirúrgica ha sido considerada en base a la confección de dos accesos laterales, uno anterior al septum y otro posterior al mismo para posteriormente descolar la membrana sinusal en ambos accesos (Betts \& Miloro, 1994). 
OLATE, S.; POZZER, L.; LUNA, A. H. B.; MORAES, M.; MAZZONETTO, R.; MOREIRA, R. W. F. \& ALBERGARIA-BARBOSA, J. R. Septum en seno maxilar. Un estudio de 91 cirugías consecutivas de elevación de piso sinusal. Int. J. Morphol., 29(4):1219-1222, 2011.

OLATE, S.; POZZER, L.; LUNA, A. H. B.; MORAES, M.; MAZZONETTO, R.; MOREIRA, R. W. F. \& ALBERGARIA-BARBOSA, J. R. Septum on maxillary sinus. A study in 91 consecutive surgeries of maxillary sinus floor elevation. Int. J. Morphol., 29(4):1219-1222, 2011.

SUMMARY: Sinus lift has been widely studied and is highly successful. The aim of this research was to establish the prevalence of intrasinusal septum in consecutive surgeries for maxillary sinus floor elevation and identify the relation with sinus membrane rupture. Ninety one surgeries were realized in patients with an indication for dental implant installation in the posterior maxilla; the patient with complete clinical and medical record (card, radiography, etc.) and without contraindications for the procedure was included in the study; the surgery was realized with local or general anesthesia and the material used for inlay graft was autogenous bone or biometarials. The septum was identified by panoramic image in the preoperatory and clinically at the time of surgery; any complication was studied and related to intrasinusal septum; the statistical study was carried out with Fisher test with $p$ value $<0.05$. Surgery was performed on 72 subjects ( 53 female and 19 male) with a mean age of 44.5 years of age; in $74.7 \%$ of patients no complications were observed, while $18.7 \%$ presented sinusal membrane rupture. The septum was observed in $18.7 \%$ (16 maxillary sinus), showing 12 septum in anterior position and 4 in posterior position; the presence of septum was positively related to membrane rupture $(\mathrm{p}<0.05)$. Finally, we can state that the intrasinusal septum was observed in $17 \%$ and was related to intraoperatory complications as membrane rupture.

KEY WORDS: Maxillary sinus; Maxillary septum; Osseous reconstruction.

\section{REFERENCIAS BIBLIOGRÁFICAS}

Beltrán, V. V. J.; Fuentes, F. R.; Engelke, W.; Marchesani, C. F. \& Flores, V. M. Localización del septo del seno maxilar a través de abordaje endoscópico. Reporte de caso y revisión de la literatura. Int. J. Morphol., 29(2):636-43, 2011.

Betts, N. \& Miloro, M. Modification of the sinus lift procedure for septa in the maxillary antrum. J. Oral Maxillofac. Surg., 52(3):332-3, 1994.

Block, M. \& Kent, J. Sinus augmentation for dental implants: the use of autogenous bone. J. Oral Maxillofac. Surg., 55(11):1281-6, 1997.

Boyne, P. J. \& James, R. A. Grafting of the maxillary sinus floor with autogenous marrow and bone. J. Oral Surg., 38(8):613-6, 1980.

Ella, B.; Noble, R. da C.; Lauverjat, Y.; Sédarat, C.; Zwetyenga, N.; Siberchicot, F. \& Caix, P. Septa within the sinus: effect on elevation of the sinus floor. Br. J. Oral Maxillofac. Surg., 46(6):464-7, 2008.

Jang, H. Y.; Kim, H. C.; Lee, S. C. \& Lee, J. Y. Choice of graft material in relation to maxillary sinus width in internal sinus floor augmentation. J. Oral Maxillofac. Surg., 68(8):1859-68, 2010.

Kim, Y. K.; Kim, S. G.; Park, J. Y.; Yi, Y. J. \& Bae, J. H. Comparison of clinical outcomes of sinus bone graft with simultaneous implant placement: a 4-month and 6-month final prosthetic loading. Oral Surg. Oral Med. Oral Pathol. Oral Radiol. Endod., 111(2):1649, 2011 .

Krennmair, G.; Ulm, C. \& Lugmayr, H. Maxillary sinus septa: incidence, morphology and clinical implications. $J$. Craniomaxillofac. Surg., 25(5):261-5, 1997.

Krennmair, G.; Ulm, C. W.; Lugmayr, H. \& Solar, P. The incidence, location, and height of maxillary sinus septa in the edentulous and dentate maxilla. J. Oral Maxillofac. Surg., 57(6):671-2, 1999.
Maestre-Ferrín, L.; Carrillo-García, C.; Galán-Gil, S.; PeñarrochaDiago, M. \& Peñarrocha-Diago, M. Prevalence, location, and size of maxillary sinus septa: panoramic radiograph versus computed tomography scan. J. Oral Maxillofac. Surg., 69(2):507-11, 2011.

Park, Y. B.; Jeon, H. S.; Shim, J. S.; Lee, K. W. \& Moon, H. S. Analysis of the anatomy of the maxillary sinus septum using 3-dimensional computed tomography. J. Oral Maxillofac. Surg., 69(4):10708, 2011.

Raghoebar, G. M.; Vissink, A.; Reintsema, H. \& Batenburg, R. H. Bone grafting of the floor of the maxillary sinus for the placement of endosseous implants. Br. J. Oral Maxillofac. Surg., 35(2):11925, 1997.

Rosano, G.; Taschieri, S.; Gaudy, J. F.; Lesmes, D. \& Del Fabbro, M. Maxillary sinus septa: a cadaveric study. J. Oral. Maxillofac. Surg., 68(6):1360-4, 2010.

Stover, J. D. The incidence, location, and height of maxillary sinus septa in the edentulous and dentate maxilla - Discussion. J. Oral Maxillofac. Surg., 57(6):671-2, 1999.

Underwood, A. S. An inquiry into the anatomy and pathology of the maxillary sinus. J. Anat. Physiol., 44(4):354-69, 1910.

Wheeler, S. L. Sinus augmentation for dental implants: the use of autogenous bone. J. Oral Maxillofac. Surg., 55(11):1287-93, 1997.

Zijderveld, S. A.; van der Bergh, J. P.; Schulten, E. A. \& ten Bruggenkate, C. M. Anatomical and surgical findings and complications in 100 consecutive maxillary sinus floor elevation procedures. J. Oral Maxillofac. Surg., 66(7):1426-38, 2008.

Dirección de correspondencia:

Prof. Dr. Jose Ricardo de Albergaria Barbosa

Av. Limeira 901, Caixa Postal 52 - CEP 13414-903

Piracicaba - SP

BRASIL

Recibido : 27-07-2011

Email: barbosa@fop.unicamp.br 